\title{
IMPLEMENTATION OF THE FRACTIONAL-STEP, ARTIFICIAL COMPRESSIBILITY WITH PRESSURE PROJECTION (FSAC-PP) METHOD INTO OPENFOAM FOR UNSTEADY FLOWS
}

\author{
Jesús Miguel Sánchez Gil \\ PhD Student, University of Calgary, Department of Mechanical and Manufacturing Engineering \\ Calgary, AB T2L1Y6, Canada, email: jesusmiguel.sanchezg@ucalgary.ca \\ Tom-Robin Teschner \\ lecturer, Cranfield University, Centre for Computational Engineering Sciences \\ Cranfield, Bedfordshire, MK43 OAL, United Kingdom,email: tom.teschner@cranfield.ac.uk \\ László Könözsy \\ senior lecturer, Cranfield University, Centre for Computational Engineering Sciences \\ Cranfield, Bedfordshire, MK43 OAL, United Kingdom, email: laszlo.konozsy@cranfield.ac.uk
}

\begin{abstract}
Commercial and open-source CFD solvers rely mostly on incompressible approximate projection methods to overcome the pressure-velocity decoupling, such as the SIMPLE (Patankar, 1980) or PISO (Issa, 1986) algorithm. Incompressible methods based on the Artificial Compressibility method (Chorin, 1967) lack a mechanism to evolve in time and need to be supplemented by a real time derivative through the dual time scheme. The current study investigates the implementation of the explicit dual time discretization of the Artificial Compressibility method into OpenFOAM and extends on that by applying the dual time scheme to the incompressible FSAC-PP method (Könözsy, 2012). Applied to the Couette $2 D$ flow at $R e=100$ and $R e=1000$, results show that for both methods accurate time evolutions of the velocity profiles are presented, where the FSAC-PP methods seemingly produces smoother profiles compared to the AC method, especially during the start-up of the simulation.
\end{abstract}

Keywords: Computational Fluid Dynamics, Incompressible Flows, Dual-Time Stepping, OpenFOAM

\section{Introduction}

Commercial software such as ANSYS Fluent as well as open-source software such as OpenFOAM rely on pressure-correction methods to solve incompressible flows. The most well-known methods are the SIMPLE family developed by (Patankar, 1980). Additionally, OpenFOAM has the PIMPLE algorithm which combines SIMPLE and PISO (Issa, 1986) solvers. All of these incompressible solvers calculate the pressure field by solving a pressure-Poisson equation, hence capturing the elliptic features of the flow field and neglecting the time-dependent changes in the pressure. As an alternative, the AC and FSPP method offer a different approach to the conventional incompressible solvers. The first method developed by Chorin (Chorin, 1967) uses a hyperbolic system of equations by introducing the time derivative in what is known as the perturbed continuity equation. This solver is solved in pseudo-time in which the derivatives vanish once the solution is converged. The second method developed by Chorin and Téman (Chorin, 1968; Témam, 1969) solves the governing equations by decomposing the velocity 
field (Helmholtz-Hodge decomposition (Ladyzhenskaya, 1963)) fractional-steps in which a pressurePoisson equation is used to project the pressure field into the next time step. Finally, a unified concept developed by Könözsy (Könözsy, 2012) was proposed to improve the convergence rate with respect to its counterpart as well as the accuracy. Therefore, the aim of this project is to implement the AC, FS-PP and FSAC-PP methods in OpenFOAM to assess the performance of each method, taking into account the restrictions given in the framework of the open-source software. Furthermore, the use of first- and second-order temporal and spatial schemes is an open area of research where the features of using these interpolations schemes can be studied and compared to the previous studies using high-order interpolation methods such as third-order Upwind, fifth MUSCL and WENO schemes (Tsoutsanis et al., 2015).

\section{Numerical Modelling}

The AC and FS-PP methods propose different techniques to uncouple the pressure field from the velocity field. The first method adds a pseudo-transient pressure term to the so-called perturbed continuity equation (Eq. 1). The second method solves the governing equation in two steps relying on the Helmholtz-Hodge decompositions. In the first step, the governing equations are solved in time without considering the pressure gradient whereas in the second step the pressure-Poisson equation is used to calculate the pressure field, which is in charge of capturing the elliptic phenomena of the pressure field. Opposite to the first method, the FS-PP method does not take into account the time changes in pressure. Könözsy (Könözsy, 2012) proposed a unified concept of both solvers called the FSAC-PP method by adding the perturbed continuity equation to the momentum equation of the first fractional step as shown below:

$$
\begin{gathered}
\frac{1}{\beta} \frac{\partial p}{\partial \tau}=-\nabla \cdot \boldsymbol{u}, \\
\frac{\widehat{\boldsymbol{u}}-\boldsymbol{u}}{\Delta \tau}=-\frac{\partial \boldsymbol{u}^{(n, m)}}{\partial t}-\nabla(\boldsymbol{u} \otimes \boldsymbol{u})^{(n)}+\nabla^{2} \boldsymbol{u}^{(n)} .
\end{gathered}
$$

As observed, the first fractional step adopts the pseudo-time approach to solve the system of the equations as well as to solve the unsteady solution by performing the dual-time step procedure. The second fractional-step is analogous to its separate counterpart, although the pressure-Poisson equation is updated in pseudo-time, as seen below:

$$
\nabla^{2} p^{(n+1)}=\frac{\rho}{\Delta \tau} \nabla \cdot \widehat{\boldsymbol{u}}
$$

After solving Eq. 3, the velocity field is updated in the same manner as in the FS-PP method, which is given by the following expression.

$$
\boldsymbol{u}^{(n+1)}=\widehat{\boldsymbol{u}}-\frac{\Delta \tau}{\rho} \nabla p^{(n+1)}
$$

Focusing on the second fractional-step, Könözsy (Könözsy, 2012) states that the introduction of the pressure-Poisson equation smoothes the system which stabilizes the calculation of the pressure. Furthermore, the Poisson solver does not require to reach a maximum number of iterations, as needed for the FS-PP method. Könözsy (Könözsy, 2012) establishes that usually ten iteration is enough to have a good enough initial estimate of the solution using the Gauss-Seidel SOR solver. This is due to the inclusion of the perturbed continuity equation which gives an initial pressure through the inclusion of 
the pseudo-time derivative. As a result, a drop in the convergence time has been achieved, according to Könözsy and Drikakis (Könözsy et al., 2012, 2014).

\section{Methodology}

In this section, the methodology followed in this study will be thoroughly described. First of all, an overview of the numerical treatment chosen to implement the equations into OpenFOAM will be explained. Secondly, the implementation of such models will be covered in Section 3.3.

\subsection{Spatial Discretization Scheme}

In previous studies using the FSAC-PP method, Könözsy and Drikakis. (Könözsy, 2012; Könözsy et al., 2012, 2014) used Characteristic-Based Methods (CB) to discretized the spatial terms of the FSACPP method. Furthermore, In (Tsoutsanis et al., 2015), a study was carried out on the implementation of several discretization scheme in structured and unstructured grids for compressible and incompressible flows. The authors used third-order Upwind, fifth-order MUSCL and fifth- and ninth-order WENO schemes for spatial extrapolation. The authors in (Smith et al., 2017) also studied the accuracy of Riemann Solvers (HLL, HLLC and Rusanov) applied to the framework of the FSAC-PP method. Finally, Teschner (Teschner et al., 2016, 2018) extended the work on CB methods to develop a MCB (Multidirectional Characteristic-Based) methods for the FSAC-PP method. However, this study is constrained by the first and second-order discretization schemes provided in OpenFOAM, whose information can be found in the user guide. The Central Difference (CD) will be used in the pressure gradient and diffusive terms whereas first- and second-order Upwind (UP) schemes will be used in the divergence term of the perturbed continuity equation and the convective term respectively.

\subsection{Time Discretization Schemes}

The explicit dual time method, in semi-discretized, first-order form is given as

$$
\frac{u^{n+1}-u^{n}}{\Delta \tau}+\frac{u^{n, m}-u^{m-1}}{\Delta t}=R\left(u^{n}\right)
$$

Here, $\boldsymbol{R}(\boldsymbol{u})$ represents the right-hand side of the Momentum equation and is evaluated based on velocities from the solution of the previous timestep or initial conditions. A fully implicit discretization would require a linearization of the residuals, resulting in the Jacobian of the system being added to the diagonal of the coefficient matrix. The first time derivative in real time is marched in pseudo time $(\tau)$ until a steady state is achieved, at which point it will vanish and only the second, real time derivative $(t)$ will be retained. The velocity component $\boldsymbol{u}^{\boldsymbol{n}, \boldsymbol{m}}$ is updated after each pseudo time iteration (inner loop) while $\boldsymbol{u}^{\boldsymbol{m}-1}$ remains constant and is updated at the beginning of a new pseudo time step (outer loop). Instead of a first order backward in time, a second order backward scheme is implemented and compared as well, which is given by

$$
\frac{3 u^{n+1}-4 u^{n}+u^{n-1}}{2 \Delta \tau}+\frac{3 u^{n, m}-4 u^{m-1}+u^{m-2}}{2 \Delta t}=R\left(u^{n}\right)
$$




\subsection{Implementation of the FSAC-PP method (fsacppFoam)}

This section will focus on the implementation of the governing equations of the FSAC-PP method using the above-mentioned numerical methods into the OpenFOAM framework. OpenFOAM relies on the popular SIMPLE, PISO algorithms as well as in a solver that combines both called PIMPLE algorithm. The source codes of these incompressible solvers are available inside the folder \$FOAM_APP/solvers. Therefore, the main structure of the solver code has been used and modified according to the requirements of the AC, FS-PP and FSAC-PP. The three solvers consist of a common file and folder structure which is explained in the studied carried out in (Sánchez Gil, 2020). The implementation of the FSAC-PP method is a combination of both AC and FS-PP codes. The dual-time stepping procedure is used to solve the governing equation in the FSAC-PP algorithm. Furthermore, the pressure and velocity field are calculated in two steps as in the FS-PP method. In the first step, the perturbed continuity from the AC method equation and the momentum equation used in the FS-PP method is solved in pseudo-time. In the second step, the pressure-Poisson equation and the updated velocity field are also solved in pseudo-time using the same methodology. The time-step is also calculated based on the CFL number. Details about the implementation can be found in (Sánchez Gil, 2020). Thus, the FSAC-PP method is implemented as follows:

1. Initialization of $U, p, U d t 1, U d t O$ and $U d t m l$ (when explicit Backward scheme is used for the real time derivative).

2. Update $U d t O$ and $U d t m l$ every real time iteration.

3. Update the fluxes $\phi$ every pseudo-time iteration and calculate the time-step based on the CFL number.

4. Update $U d t 1$ to the converged solution $U$ in pseudo-time and solve for the real time derivative.

5. Solve explicitly the perturbed continuity equation (Eq. 1) for the pressure and update it at the boundaries.

6. Solve semi-implicitly the momentum equation without the pressure gradient term for the intermediate velocity field.

7. Solve semi-implicitly the pressure-Poisson equation until reaching the maximum number of nonorthogonal correction iterations, if required.

8. Explicitly update the velocity using the intermediate velocity field and pressure gradient results.

9. Repeat steps 2-8 until reaching convergence in pseudo-time. Then, start next real time step until the final time is reached.

\section{Results and discussion}

In this section, the AC, FS-PP and FSAC-PP codes implemented into OpenFOAM will be validated by running simulations of a Couette flow case at Reynolds number equal to 100 and 1000 and comparing the theoretical evolution of the velocity profile with the approximated solution obtained from the incompressible models. Prior to this analysis, Sánchez (Sánchez Gil, 2020) performed a grid convergence study as well as a parametric study of the influence of CFL number in the solution. The results have reached grid independence with a fine-grid grid convergence index (GCI) of GCI=3e-4. Since this case has an analytical solution available, we are able to measure the absolute error. We measure the velocity at the centerline (which is the average between the upper and lower wall once the solution has reached a steady state) and measured an absolute error of $5.68 \mathrm{e}-6 \mathrm{~m} / \mathrm{s}$ which corresponds to a relative error of $1.14 \mathrm{e}-3 \%$. These results suggest that our results will be independent of the grid we 
are using. Furthermore, initial studies suggest that the optimal CFL number for this case is 0.8 , as it provided the same accuracy as with lower CFL number at a faster convergence rate.

The results in Fig. 1 show the comparison between the analytical evolution of the velocity profile in time with the approximated solution obtained from the AC, FS-PP and FSAC-PP methods when using different time schemes (BE: Explicit Backward -Implicit Euler; BB: Explicit Backward - Implicit Backward; EE: Explicit Euler - Implicit Euler).
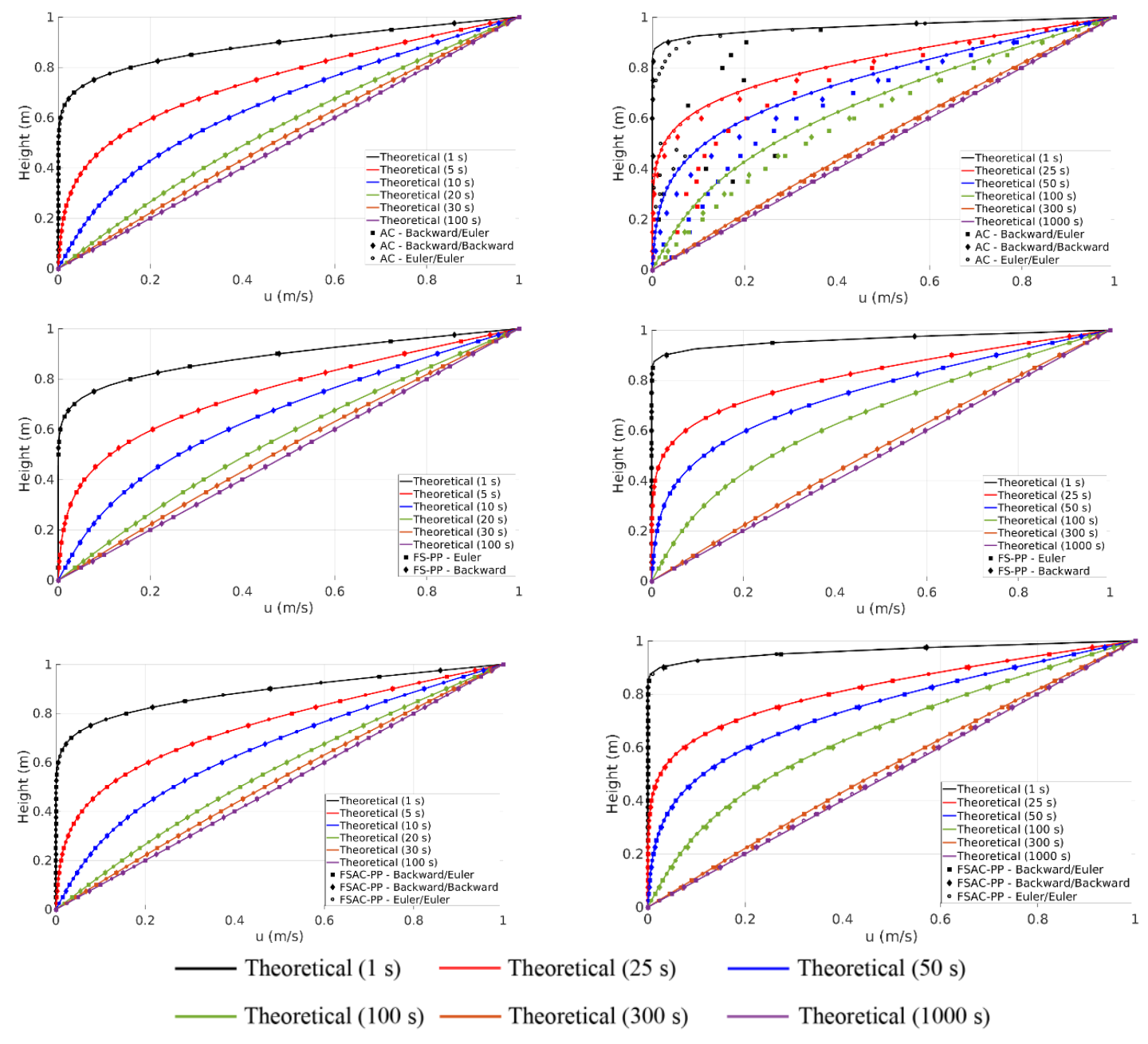

- Backward/Euler $\diamond$ Backward/Backward o Euler/Euler

Figure 1. Evolution of the velocity profiles in time using the AC (top), FS-PP (middle) and FSAC$P P$ (bottom) method for $R e=100$ (left) and $R e=1000$ (right).

This study is constrained by the first- and second-order time schemes provided in OpenFOAM to advance the solution in pseudo- and real-time, opposite to the approaches taken in (Könözsy et al, 2012, 2014; Teschner et al. 2019) in which the authors use a multistage explicit fourth- and third-order RungeKutta scheme, respectively, to advance the solution in time. However, the first author suggests that an 
explicit time scheme from first- up to a higher-order of accuracy to advance the solution in real time can be used. Furthermore, the solution of the velocity profile at $\mathrm{Re}=100$ shows that the velocity profile accurately evolves through time. No substantial change is observed when using the different time scheme set-ups with respect to accuracy. In the study carried out by (Sánchez Gil, 2020), the absolute and relative errors tables are shown for each time scheme set-up. These show that the accuracy of the models is between $10^{-4}$ and $10^{-5}$. Focusing on $t=100 \mathrm{~s}$ when the solution has become stationary, the results shows that the solutions using the second-order time schemes BB demonstrate to be more accurate than BE or EE which is a fully first-order time scheme set-up. Given the results, it can be stated that the AC, FS-PP and FSAC-PP method can be used with the provided numerical method in OpenFOAM which have lower order of accuracy than those use in (Teschner et al., 2018, 2019) and (Könözsy et al., 2012, 2014). The Couette flow has been also simulated for $\mathrm{Re}=1000$ for this case. In this case, the kinematic viscosity is smaller than before, playing a less relevant role in the simulation and being the main reason why the velocity profile develops at a slower rate, as observed in Figure 1 on the right. The results show that the absolute and relative errors of the average velocity have the same trend as at $\operatorname{Re}=100$, when comparing the steady-state solution at $\mathrm{Re}=1000$. However, the AC method shows oscillatory behavior when evolving the solution in time. The AC method can show oscillation in the results mainly caused by the instabilities generated by the pseudo-pressure waves in the domain (Chorin, 1967). This can be smoothed by changing the beta parameter which acts like a smoothing parameter and also speeds the convergence of the solution. However, this parameter was set equal to 1 for all the cases for the sake of uniformity. A parametric study is suggested to assess if the oscillation vanish by changing this parameter. Also, the first-order set-up (EE) correctly predicts the time evolution, meaning that the additional dissipation introduced by this discretization schemes may help to smooth the oscillations.

\section{Summary}

In this study, we have shown the implementation of the dual time scheme into OpenFOAM through an explicit time discretization in real time. Applied to the Couette problem at $\operatorname{Re}=100$ and $\operatorname{Re}=1000$, we have shown that the elliptic features of the FSAC-PP method help to stabilize the solution, especially during start-up of the simulation. Furthermore, both approaches have shown that the correct time evolution of the velocity profiles is obtained and further investigation into the implicit dual time discretization may be conducted to increase performance.

\section{References}

[1] Chorin, A. J. (1967). A numerical method for solving incompressible viscous flow problems. Journal of Computational Physics, 2(1), 12-26. https://doi.org/10.1016/0021-9991(67)90037-X

[2] Chorin, A. J. (1968). Numerical solution of the Navier-Stokes equations. Computational Fluid Mechanics, 22 Academic Press, Inc. (pp. 17-34). https://doi.org/10.1016/B978-0-12-174070-2.50005-8

[3] Issa, R. I. (1986). Solution of the implicitly discretised fluid flow equations by OperatorSplitting. Journal of Computational Physics, 62(1), 40-65. https://doi.org/10.1016/0021-9991(86)90099-9

[4] Könözsy, L. (2012). Multiphysics CFD modelling of incompressible flows at low and moderate Reynolds numbers. Cranfield University. 
[5] Könözsy, L. and Drikakis, D.: A coupled high-resolution fractional-step artificial compressibility and pressure-projection formulation for solving incompressible multi-species variable density flow problem at low Reynolds numbers. ECCOMAS 2012 - European Congress on Computational Methods in Applied Sciences and Engineering, e-Book Full Papers. Vienna, Austria, pp. 3206-3220.

[6] Könözsy, L. and Drikakis, D. (2014). A unified fractional-step, artificial compressibility and pressure-projection formulation for solving the incompressible Navier-Stokes equations. Communications in Computational Physics, 16(5), 1135-1180. https://doi.org/10.4208/cicp.240713.080514a

[7] Ladyzhenskaya, O. A. (1963). The mathematical theory of viscous incompressible flow. New York: Gordon and Breach.

[8] Patankar, S. V. (1980). Numerical heat transfer and fluid flow. Taylor \& Francis (2 $2^{\text {nd }}$ ed).

[9] Sánchez Gil, J. M. (2020). Implemenation of the fractional-step, artificial compressibility with pressure projection (FSAC-PP) method into openFOAM for steady and unsteady simulations. Cranfield University.

[10] Smith, K., Teschner, T.-R. and Könözsy, L.: On approximate riemann solvers within the concept of the unified fractional-step, artificial compressibility and pressure projection method. 2017 MicroCad International Multidisciplinary Scientific Conference. Miskolc: MultiScience. https://doi.org/10.26649/musci.2017.062

[11] Témam, R. (1969). Sur l'Approximation de la Solution des Équations de Navier-Stokes par la Méthode des Pas Fractionnaires (I). Archive for Rational Mechanics and Analysis, 32(2), 135 153. https://doi.org/10.1007/BF00247678

[12] Teschner, T.-R., Könözsy, L. and Jenkins, K. W.: Numerical investigation of an incompressible flow over a backward facing step using a unified fractional-step, artificial compressibility and pressure-projection (FSAC-PP) method. 2016 MicroCad International Multidisciplinary Scientific Conference. Miskolc: MultiScience. https://doi.org/10.26649/musci.2016.096

[13] Teschner, T.-R., Könözsy, L. and Jenkins, K. W. (2018a). A three-stage algorithm for solving incompressible flow problems. 2018 MicroCad International Multidisciplinary Scientific Conference. Miskolc: MultiScience. https://doi.org/10.26649/musci.2017.061

[14] Teschner, T. R., Könözsy, L. and Jenkins, K. W. (2018b). Predicting non-linear flow phenomena through different characteristics-based schemes. Aerospace, 5(1).

https://doi.org/10.3390/aerospace5010022

[15] Teschner, T. R., Könözsy, L. and Jenkins, K. W. (2019). A generalised and low-dissipative multidirectional characteristics-based scheme with inclusion of the local Riemann problem investigating incompressible flows without free-surfaces. Computer Physics Communications, 239 Elsevier B.V. (pp. 283-310). https://doi.org/10.1016/j.cpc.2018.07.026

[16] Tsoutsanis, P., Kokkinakis, I. W., Könözsy, L., Drikakis, D., Williams, R. J. R. and Youngs, D. L. (2015). Comparison of structured- and unstructured-grid, compressible and incompressible methods using the vortex pairing problem. Computer Methods in Applied Mechanics and Engineering, 293, 207-231. https://doi.org/10.1016/j.cma.2015.04.010 